\title{
Effet de la mouillabilité d'un film polymère sur les températures et l'action nématicide obtenues en solarisation d'un sol de culture
}

\author{
A. Jaffrin 1, S. Makhlouf 1, C. Scotto La Massese 2, A. Bettachini 2 et R. Voisin 2 \\ 1 CNRS, laboratoire écothermique, et laboratoire URIH-INRA, route des Colles, ZA Sophia-Antipolis, 06410 Biot; \\ 2 INRA, centre Antibes, station de recherches de nématologie; B.P. 2078, 06606 Antibes Cedex, France
}

(reçu le 25 août 1988, accepté le 27 avril 1989)

\begin{abstract}
Résumé - On a examiné le résultat de la désinfection d'un sol de culture sous l'effet, exclusif ou combiné, d'un réchauffement par rayonnement solaire sous un film polymère de type anti-buée et d'un traitement au bromure de méthyle. Les mesures thermiques, confirmées par des calculs, montrent qu'un tel film, grâce à sa bonne transmittivité optique et à sa propriété de barrière infrarouge en présence de condensation, est capable d'assurer une très forte montée en température de la surface du sol. La comparaison avec le sol d'une parcelle témoin montre qu'il suffit alors d'un apport modique de biocide pour assurer l'élimination d'organismes résiduels indésirables (adventices et nématodes). II a été toutefois constaté que la mouillabilité de ce film était limitée à quelques semaines et que les propriétés optiques qui lui étaient associées disparaissaient avec elle.
\end{abstract}

film polymère - mouillabilité - transmission optique - température de sol - solarisation - désinfection - nématodes

Summary - The wettability of a plastic mulch. Its effects on the temperatures and its nematicide action during solarization of a cultivated soil. The solarization of a soil under a highly wettable transparent plastic mulch, with or without addition of methyl bromide, gives grise to particularly high soil surface temperatures. A numerical simulation shows that this is due to the high optical transmittance, combined with the efficient infra-red barrier property of the wetted film. Under such conditions, the comparison with a bare portion of soil shows that a moderate use of fumigant is enough to get rid of the residual undesirable organisms (weeds or nematodes). It must be noted, however, that the good optical properties of the film disappear when its wettability stops, which happens after a few weeks of exposure.

polymer film - wettability - optical transmittance - soil temperature - solarization - disinfection - nematodes

\section{INTRODUCTION}

Les techniques de désinfection des sols agricoles contaminés par des agents pathogènes mettent traditionnellement en jeu des traitements thermiques par vapeur d'eau surchauffée, jugés coûteux, ou des traitements chimiques qui peuvent laisser des résidus inacceptables dans les produits consommables et imposent souvent une couverture du sol par des films peu perméables au gaz injecté. Aussi, les solutions permettant de diminuer les consommations énergétiques des traitements thermiques ou de réduire les taux d'utilisation de produits chimiques font-elles actuellement l'objet de nombreuses études.
La solarisation des sols sous film plastique, en particulier, est de plus en plus utilisée depuis quelques années, pour provoquer la montée en température nécessaire à l'élimination de diverses formes d'agents phytopathogènes telluriques ainsi que des mauvaises herbes. Une bibliographie des études consacrées à ce sujet depuis 10 ans a été faite par Katan et al. (1987). On utilise l'action combinée de l'accroissement de la teneur en humidité du sol bâché préalablement arrosé, qui déclenche la remise en activité des organismes vivants, et de l'élévation de la température jusqu'à un niveau léthal (au dessus de $43,5^{\circ} \mathrm{C}$ ) à l'issue d'une période de réchauffement par rayonnement solaire. Des résultats satisfai- 
sants ont ainsi été obtenus sous des latitudes favorables, comme celles d'Israël (Katan et al., 1976), de Californie (Johnson et al., 1981), de Floride (McSorley et Parrado, 1986), de Crète (Malathrakis et al., 1983), d'Australie (Kassaby, 1985) ou de Corée (Kye et Kim, 1985).

L'essai sous les latitudes du sud de l'Europe, au climat moins favorable à ce mode de désinfection, a donné lieu à des expériences de solarisation de sol sous serre en Italie (Garibaldi, 1983), en Allemagne Fédérale (Braun et al., 1987) et en France (Goisque et al., 1984), et, en particulier, à des essais multiples aux stations de I'INRA d'Antibes et de Fréjus (sud-est de la France), de 1979 à 1982, aussi bien sous serre qu'en plein air (Berninger et al., 1985). On a pu constater qu'il fallait choisir de préférence des films dotés de bonnes propriétés thermiques (films $\mathrm{PE}$ alvéolaires, films EVA ou, mieux, PVC) pour compenser le handicap du climat. Dans certaines conditions, l'élévation en température de la couche superficielle du sol a été suffisante pour provoquer une réduction significative de la population de nématodes et de la mycoflore jusqu'à $40 \mathrm{~cm}$ de profondeur, et cela sans affecter notablement les populations bactériennes; on peut donc espérer un résultat comparable à ce qui est enregistré dans les pays chauds, à condition de bénéficier de conditions climatiques estivales favorables et d'éviter la prolifération intempestive de mauvaises herbes sous le film en début de traitement.

En 1984, de nouveaux essais, conduits au centre de I'INRA d'Antibes de fin juillet à septembre, ont permis de mettre en évidence le bénéfice que l'on pouvait tirer de l'usage de certains films plastiques à forte mouillabilité (Scotto la Massese, 1984): la transmission optique du film n'étant plus affectée par le dépôt de condensation en gouttes disjointes sur sa face interne, un tel film devient aussi performant globalement qu'un film PE alvéolaire (qui offre pourtant, de nuit, une meilleure isolation), tout en étant beaucoup moins encombrant et moins coûteux. Une remarque dans le même sens a été faite par Avissar et al; (1986) sur des films de polyéthylène usagés dont les propriétés de surface avaient évolué. L'expérience a été reprise à Antibes, du 20 juin au 30 août 1985, dans le but d'optimiser la montée en température des parcelles par un meilleur choix de la période d'insolation, avec pour seul matériau de couverture un film doté de propriétés de forte mouillabilité. L'action complémentaire d'un agent chimique biocide gazeux (bromure de méthyle) à demi-dose a été étudiée en parallèle sur des parcelles séparées, avec l'intention de réduire le développement éventuel d'adventices sous le film. Les résultats observés sur le plan thermique et nématologique à l'issue de cette dernière expérimentation sont présentés ci-dessous.

\section{DISPOSITIF EXPÉRIMENTAL}

Un dispositif de blocs de Fisher comportant 4 répétitions de $9,7 \mathrm{~m}^{2}(2,3 \mathrm{~m} \times 4,2 \mathrm{~m})$ de superficie, séparés par des allées de $0,6 \mathrm{~m}$, a été mis en place pour une culture d'anémones (Anemone coronaria L.) sur un sol initialement infesté par Colletotrichum acutatum:

- une parcelle A témoin, non traitée et laissée à l'air libre;

- une parcelle $B$, traitée préalablement par $80 \mathrm{~g} / \mathrm{m}^{2}$ de $\mathrm{CH}_{3} \mathrm{Br}$, et laissée ensuite à l'air libre;

- une parcelle $C$, soumise à solarisation sous un film EVA transparent mouillable, et non traitée chimiquement;

- une parcelle $D$, soumise à solarisation sous un film identique à $\mathrm{C}$, mais traitée préalablement avec $40 \mathrm{~g} / \mathrm{m}^{2}$ de $\mathrm{CH}_{3} \mathrm{Br}$.

Chaque parcelle a été initialement bêchée et a reçu un bassinage de 40 I d'eau au $\mathrm{m}^{2}$. Puis des sondes de température, montées sur des supports, ont été enfouies dans le sol. Le film utilisé en paillage des parcelles soumises à solarisation, d'une épaisseur de $50 \mu \mathrm{m}$, est à base de copolymère éthyl-vynil-acétate, doté d'un agent lui donnant une plus faible perméabilité gazeuse et lui conférant, en outre, une forte mouillabilité vis-à-vis de l'eau (film désigné ici EP). Le film EP a été déployé à la surface de 2 des parcelles le 20 juin et enlevé le 30 août, date de mise en place de la culture d'anémones.

Les sondes de température mises en place sont des circuits intégrés $A D 590$, à réponse linéaire en courant, ajustées à l'intérieur de cylindres de cuivre étanches de $25 \mathrm{~mm}$ de long et $6 \mathrm{~mm}$ de diamètre, conçus pour un meilleur contact thermique avec le sol environnant. La distance mutuelle des cylindres sur une même verticale est assurée, à la précision du $\mathrm{mm}$, par un support de PVC rigide (d'une épaisseur de $5 \mathrm{~mm}$ ), moins conducteur que le sol, percé d'une série de trous calibrés d'axes horizontaux. La sonde de surface est en contact avec le sol et reçoit le rayonnement sur sa face supérieure. Son absorption dans le visible est comparable à celle du sol $(\alpha \approx 0,8)$. La mise en place a été faite après le bassinage, pour éviter une variation de la position des sondes par rapport à la surface du sol.

Des valeurs instantanées des températures sont enregistrées toutes les $h$ sur cassette magnétique par un système d'acquisition CNRS, 
piloté par un micro-processeur M6802, d'une autonomie de $30 \mathrm{j}$.

\section{COMPORTEMENT THERMIQUE OBSERVÉ POUR LE SOL SOUS PAILLAGE}

L'examen des courbes de température des sondes de surface, relevées sous film plastique EP (Fig. 1 et 2), fait apparaître des maximums remarquablement élevés (situés respectivement au voisinage de $75^{\circ} \mathrm{C}$ et de $85^{\circ} \mathrm{C}$, sur les deux parcelles $C$ et $D$, soumises à solarisation sous film) pendant une longue succession de journées très ensoleillées. Ces valeurs sont bien supérieures à ce qui est usuellement observé sous un film de polyéthylène ordinaire: c'est une manifestation évidente de l'excellente transmittivité optique du film EP, en présence de condensation, lorsqu'il est mouillable.

Une deuxième observation importante est la chute subite des températures sous paillage au $45^{\mathrm{e}}$ jour d'exposition, simultanément constatée sur les deux parcelles $C$ et $D$ (Fig. 2 et 3 ). A partir de ce stade, les valeurs obtenues redeviennent voisines de ce qui est observable sous un

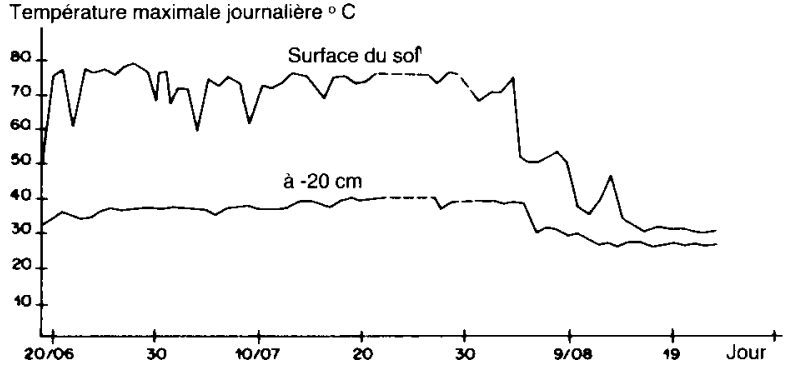

Fig. 2. Evolution sur l'ensemble de la période de solarisation des températures maximales de surface et à $20 \mathrm{~cm}$ de profondeur, observées sous film EP. On notera le changement brutal intervenu en surface à partir du 4 août et sa conséquence en profondeur.

film de polyéthylène ordinaire, comme le montre un enregistrement présenté en Figure 4, relatif à une expérimentation de juillet 1984. Cela semble donc indiquer que la qualité de la transmission optique du film EP ayant perdu sa mouillabilité relègue celui-ci au niveau d'un polyéthylène ordinaire. Cette hypothèse est confortée par les clichés $A, B$ et $C$ de la Figure 5 , qui montrent l'allure de divers échantillons de films polymères: un film EP mouillable (en début d'expérience), le même film devenu non mouillable (après
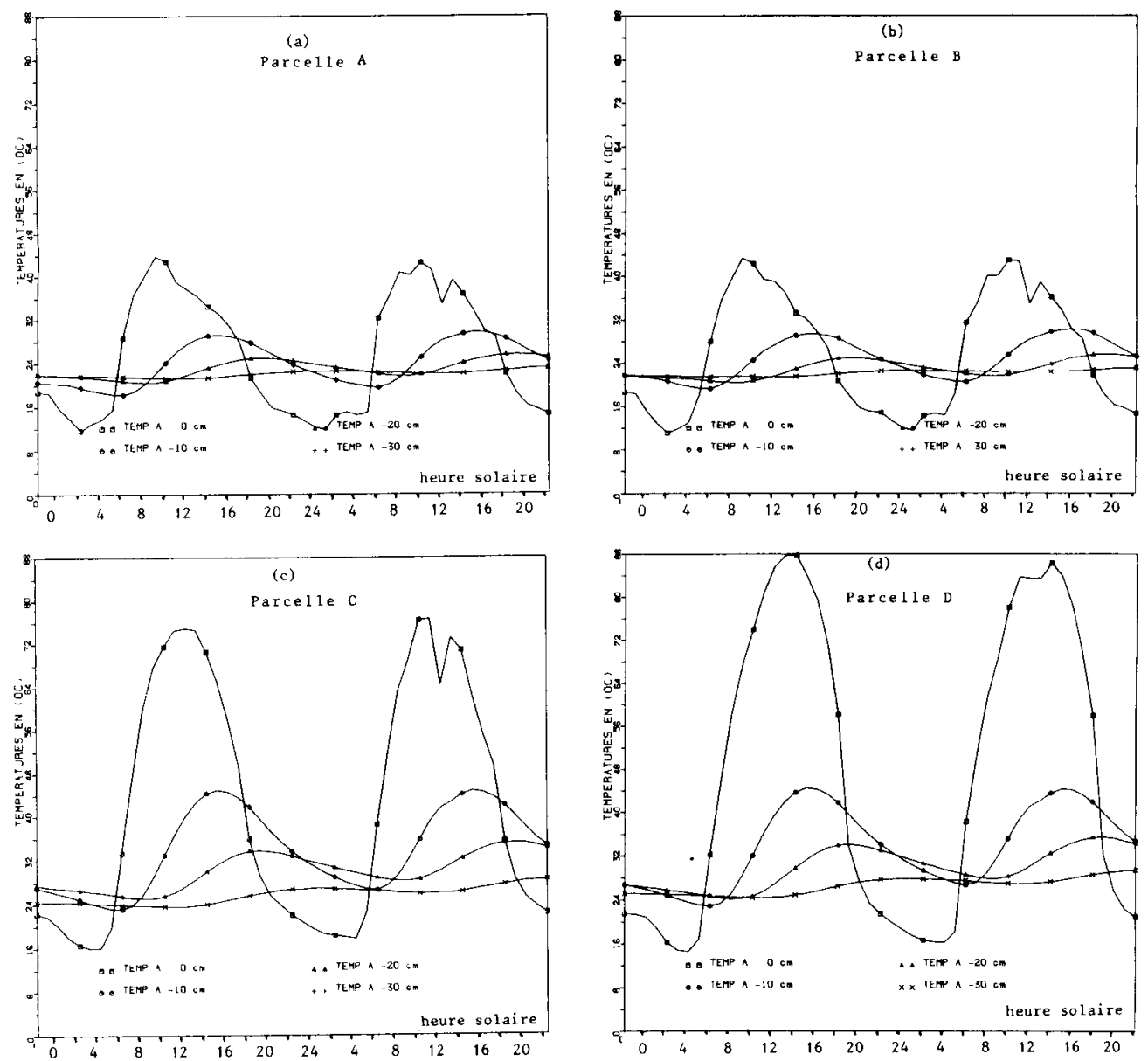

Fig. 1. Evolution journalière des températures en surface et à différentes profondeurs sur les deux parcelles de sol nu (1a et 1b), et sur les deux parcelles paillées de film EP (1c et 1d), au cours de journées caractéristiques du début de l'expérience de solarisation (20 et 21 juin 1985). 

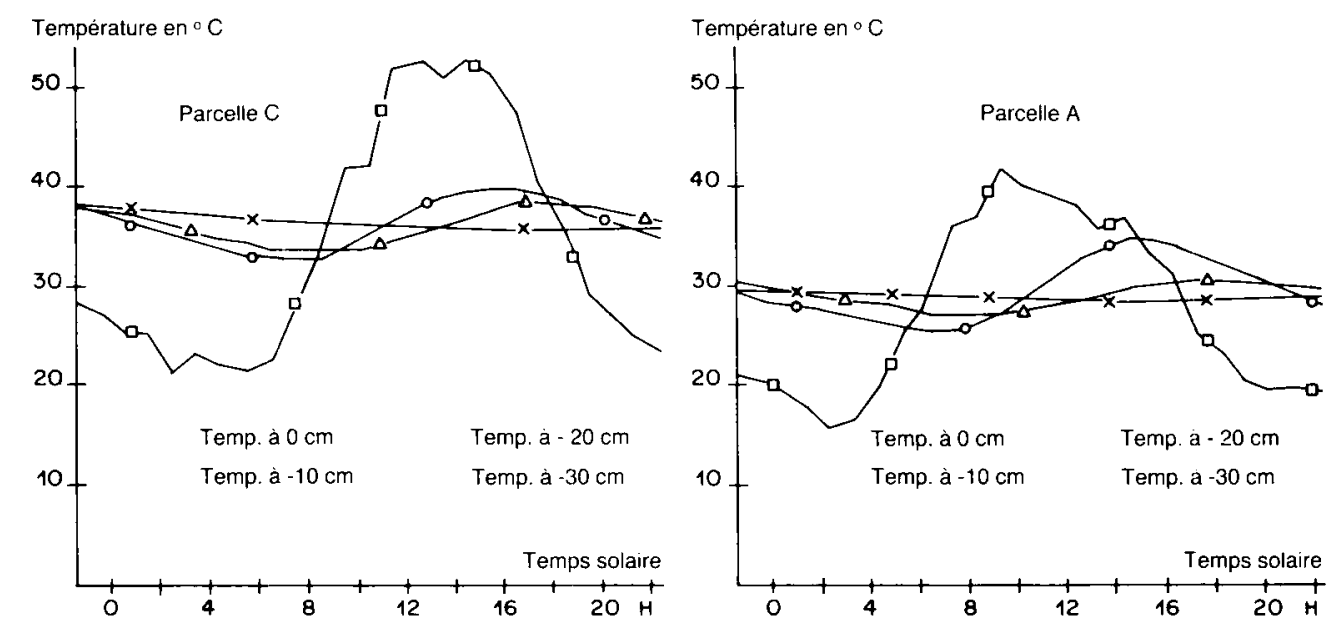

Fig. 3. Variations journalières des températures en surface et en profondeur pour la journée du 4 août 1985, pour lesquelles les films EP des parcelles paillées ont perdu leur mouillabilité (Fig. 3a). En Figure 3b, est indiqué le comportement d'une parcelle de sol sans paillage au même jour.

quelques semaines de pose) et un film de polyéthylène ordinaire (non mouillable), placé sur un sol humide.

La chute des températures, qui ne s'est pas manifestée sur les parcelles de sol nu, n'est pas imputable à un effet climatique. L'analyse des conditions météorologiques montre que le phénomène coïncide avec l'apparition, après $45 \mathrm{j}$ de temps calme, d'une journée de vent violent, qui a soumis les films à de fortes secousses et les a débarrassés de la lame d'eau jusqu'alors adhérente. Cette lame d'eau a entraîné dans sa chute les dernières traces de produit tensio-actif contenu dans le polymère traité. Les films, ainsi essorés, ont perdu leur propriété de mouillabilité. En effet, la reconstitution du dépôt de condensats s'est faite par de l'eau pure sur du film qui n'était plus constitué que de chaînes polymères classiques à radicaux hydrophobes.

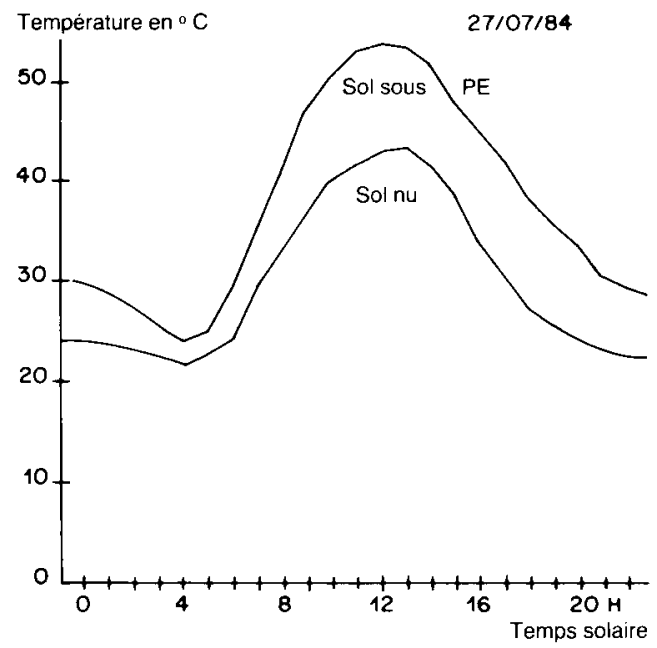

Fig. 4. Courbes typiques d'évolution journalière des températures de surface observées sur sol nu et sous paillage de film de polyéthylène ordinaire non mouillable (courbes relevées lors d'une expérimentation conduite en été 1984, pour des conditions comparables à celles de la Figure 3 ).
Le but de la discussion qui suit est de montrer que cette évolution des propriétés de surface des films suffit à expliquer l'évolution des températures. Cela sera fait en analysant le mécanisme d'échauffement du sol sous un film mouillable et sous un film non mouillable, à l'aide d'un modèle simple, pour rendre compte, en particulier, des conséquences thermique de la perte de mouillabilité d'un film.

\section{MODÉLISATION DU COMPORTEMENT THER- MIQUE D'UN SOL SOUS PAILLAGE}

Trois types de transferts thermiques sont mis en jeu ici: le rayonnement incident à travers le film, les pertes calorifiques à travers le film vers l'atmosphère et la conduction thermique vers le sol en profondeur.

\section{Les apports rayonnants}

\section{Le rayonnement solaire incident}

La modélisation du rayonnement solaire global sur un plan horizontal est fort classique et peut faire appel à la formule suivante (Perrin de Brichambaut \& Vauge, 1982):

$$
G=\left(1270-56 T_{L}\right)(\cos \Theta)\left(T_{L}+36\right) / 33
$$

où $\Theta$ est l'angle zénithal du soleil et $T_{L}$ le facteur de trouble local et saisonnier. ( $\Theta$ est utilisé ici, au lieu de l'angle usuel de hauteur du soleil, car il coïncide avec l'angle d'incidence sur le film horizontal utilisé plus bas).

La loi de variation de $\Theta$ avec la date et l'heure est donnée par la formule connue (Duffie \& Beckman, 1974):

$$
\cos \Theta=\sin \delta \sin \phi+\cos \delta \cos \phi \cos w
$$


b

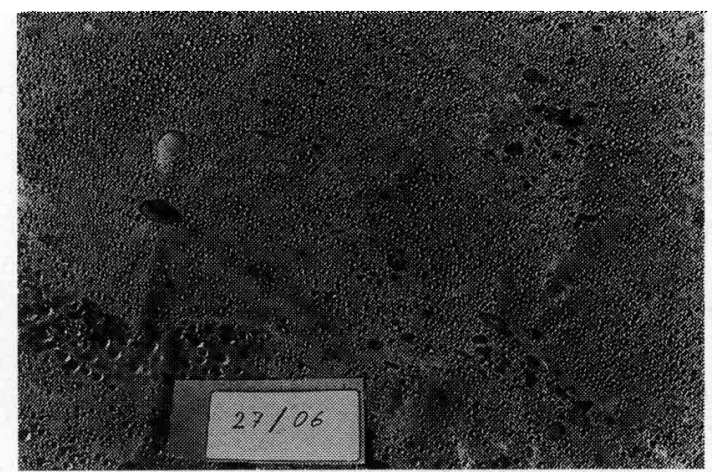

C

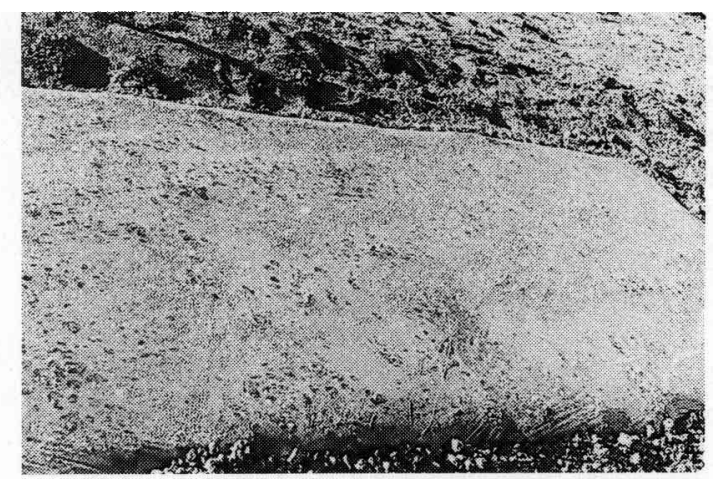

Fig. 5. Clichés montrant l'allure de divers films polymères en présence de condensation. En $5 \mathrm{a}$, le film anti-buée EP en début d'essai est intégralement nappé d'une couche d'eau de condensation en face inférieure; en $5 b$, un film de polyéthylène ordinaire manifeste un comportement hydrophobe caractéristique, avec de nombreuses gouttelettes de tailles variées; en $5 \mathrm{c}$, le film EP, une fois qu'il a perdu sa mouillabilité, présente un comportement très voisin de celui du film de polyéthylène ordinaire.

où $\delta$ est la déclinaison à la date considérée, $\phi$ la latitude du lieu et $w$ l'angle horaire.

On obtient une bonne représentation du rayonnement incident, mesurable par ciel dégagé, sur le site de l'expérience, avec une valeur de $T_{L}=5$.

\section{La contribution des réflexions partielles sur les dioptres}

La réflectivité globale d'un film, avec ou sans dépôt d'eau sur sa surface inférieure, résulte de la somme des contributions des réflexions partielles et des réflexions totales, au passage des dioptres successifs. Les réflexions partielles se produisent sur les dioptres plans air-polymère, polymère-air et polymère-eau, ainsi que sur les dioptres sphériques eau-air.

Pour la part de réflexion partielle au passage d'un dioptre,on utilise la loi classique de Fresnel (Duffie et Beckman, 1974) pour calculer l'intensité de la composante réfléchie d'un faisceau incident non polarisé:

$$
\begin{gathered}
\rho=1 / 2 \sin ^{2}\left(\Theta_{i}-\Theta_{r}\right) / \sin ^{2}\left(\Theta_{i}+\Theta_{r}\right) \\
+1 / 2 \operatorname{tg}^{2}\left(\Theta_{i}-\Theta_{r}\right) \operatorname{tg}^{2}\left(\Theta_{i}+\Theta_{r}\right)
\end{gathered}
$$

où $\Theta_{i}$ et $\Theta_{r}$ sont les angles d'incidence et de réfraction considérés, reliés par la relation de Descartes générale:

$$
n_{i} \sin \Theta_{i}=n_{r} \sin \Theta_{r}
$$

$n_{i}$ et $n_{r}$ étant les indices des deux milieux en contact.

Le franchissement de deux dioptres successifs parallèles, $\left(D_{12}\right)$ et $\left(D_{23}\right)$ donne lieu à une série infinie de rayons réfléchis entre les deux plans, et le coefficient de transmission global $T$ s'exprime, après resommation selon un processus classique (Duffie et Beckma, 1974), sous la forme connue caractérisant la lame à faces parallèles (milieu 2) séparant les milieux 1 et 3 :

$$
T=\left(1-\rho_{12}\right)\left(1-\rho_{23}\right) /\left(1-\rho_{12} \rho_{23}\right)
$$

où $\rho_{12}$ et $\rho_{23}$ s'obtiennent par les relations de Fresnel appliquées à chacun des dioptres, avec les angles d'incidence correspondants.

On peut généraliser cette approche au cas de trois dioptres successifs, qui représentent alors un film polymère mouillé d'une couche plane d'eau de condensation. Dans le cas où le milieu 4 et le milieu 1 sont identiques (l'air ambiant), le coefficient de transmission optique s'écrit:

$$
\mathrm{T}=\frac{\left[1-\rho_{12}(\Theta)\right]\left[1-\rho_{23}\left(\Theta^{\prime}\right)\right]\left[1-\rho_{31}(\Theta)\right]}{1-\rho_{12}(\Theta) \rho_{23}\left(\theta^{\prime}\right)-\rho_{13}\left[\rho_{12}(\Theta)+\rho_{23}\left(\Theta^{\prime}\right)-2 \rho_{12}(\Theta) \rho_{23}(\Theta)\right]}
$$

où $\Theta$ est l'angle d'incidence dans le milieu 1, et $\Theta^{\prime}$, l'angle d'incidence dans le milieu 2. 


\section{Calcul des réflexions totales au sein des dioptres sphériques}

Un raisonnement simple (Jaffrin \& Venard, 1982) permet d'avoir une estimation du premier ordre, applicable à un rayonnement solaire direct incident, perpendiculairement au film: les rayons incidents sur la surface de la couronne extérieure (Fig. 6) subissent une réflexion totale dans le dioptre demi-sphérique et ressortent. Le rayon interne de la couronne est déterminé par l'angle limite de réflexion totale $\phi,(\sin \phi=1 / n, n$ étant l'indice de réfraction de l'eau). On trouve ainsi que le rapport $\tau$ de la surface du cercle interne (donnant lieu à transmission) à la surface totale du cercle méridien du dioptre égale $1 / n^{2}$; cela permet de donner une première estimation du coefficient de transmission optique des gouttes (on trouve une transmission optique de $57 \%$ avec un indice de l'eau de 1,33).

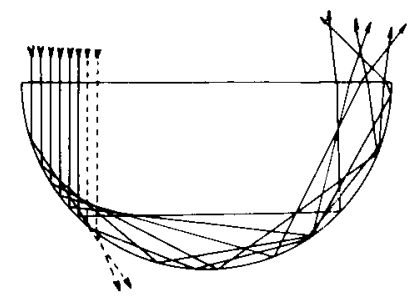

Fig. 6. Schéma des trajets optiques de rayons incidents normalement sur une lentille plan-sphérique représentant une goutte de condensation adhérente à un film non mouillable. Les rayons (en traits pleins) incidents sur la couronne périphérique du plan de la goutte subissent des réflexions totales, et sont renvoyés vers l'extérieur de façon non directive.

Pour aller plus loin, il faut tenir compte de la répartition des gouttes hémisphériques sur un film non mouillable.

- Au vu des clichés de films non mouillables, on peut considérer que les gouttes croissent de façon indépendante, sur des sites de nucléation disposés aléatoirement sur le film, pour occuper finalement "presque totalement» sa surface: des gouttes de petit diamètre viennent ainsi combler l'espace entre des gouttes de plus grand diamètre (Fig. 7). Dans ces conditions, la transmission optique globale de l'ensemble de la population de gouttes revient à celle d'une goutte individuelle et redonne:

$$
\tau=1 / n^{2} \text { soit } 57 \% \text { de transmission. }
$$

On peut généraliser ce résultat, en considérant une incidence des rayons différente de la normale au plan du film qui supporte la goutte, soit $\Theta_{i} \neq 0$; cela revient à remplacer dans les expressions précédentes

$$
n^{-2} \text { par } n^{-1}\left[n^{2}-\sin 2 \Theta_{i}\right]^{-1 / 2}
$$

comme le montre la discussion de l'annexe 1, qui donne, en outre, l'expression finale du coefficient

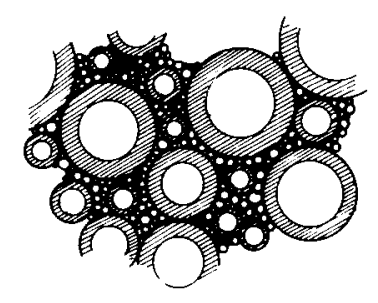

Fig. 7. Modèle pour la répartition de gouttes de condensation sur un film non mouillable; l'agencement erratique de demisphères de rayons variés conduit à un recouvrement dense de la surface du film.

de transmission optique d'un film humide non mouillable.

II est donc maintenant facile de comparer les transmissions optiques d'un même film polymère, suivant qu'il est $\mathrm{sec}$, mouillé d'un film d'eau continu ou mouillé de gouttes réparties de façon dense; c'est ce qui est présenté dans la Figure 8, où est indiqué, à titre de référence, le coefficient de réflexion à la surface d'un dioptre simple.

On constate que le film mouillé continûment a une meilleure transmission optique que le film sec, jusqu'aux angles d'incidence voisins de $60^{\circ}$; (au delà, la multiplicité des dioptres devient pénalisante). Cette transmission est comprise entre $86 \%$ et $94 \%$ pour des angles d'incidence de $60^{\circ}$ à $30^{\circ}$. Cela correspond à des hauteurs du soleil de $30^{\circ}$ à $60^{\circ}$, qui concernent l'essentiel des heures chaudes d'été, dans le cadre d'une expérience de solarisation d'un sol horizontal.

Au contraire, le même film polymère, devenu non mouillable, subit la plus forte altération de sa transmission optique pour les incidences faibles $(53 \%$ au lieu de $94 \%)$. Cette transmission s'améliore à partir de $20^{\circ}$ pour culminer à $66 \%$ aux alentours de $60^{\circ}$ d'incidence, mais le déficit par rapport à un film sec ou mouillable est encore de 20 points, alors que la part essentielle de la trajectoire du soleil en été est déjà franchie.

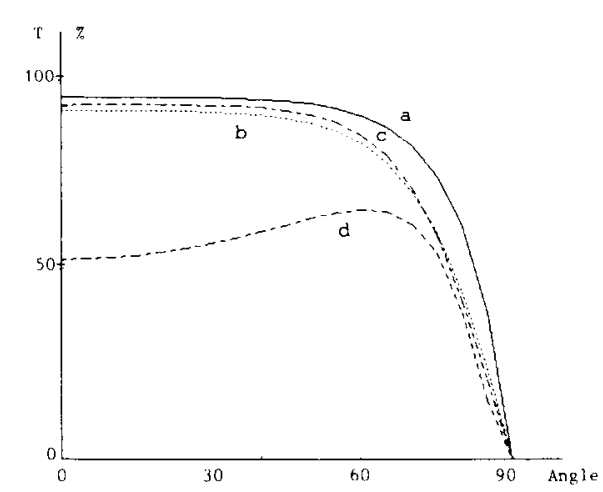

Fig. 8. Coefficient de transmission optique d'un dioptre plan (courbe a), d'un film polymère sec (courbe b), d'un film polymère mouillé continûment (courbe $c$ ), et d'un fillm couvert, en face inférieure, d'une répartition dense de gouttes (courbe d). En abscisse est porté l'angle d'incidence des rayons lumineux. L'indice de réfraction utilisé est 1,5 pour le polymère et 1,33 pour l'eau. 
Dans cette discussion, l'éventuelle réduction de transmission optique due à une salissure de surface du film a été volontairement éludée, et, d'autre part, l'absorption due au polymère luimême a été supposée négligeable aux épaisseurs considérées $(100 \mu \mathrm{m})$.

\section{Les déperditions thermiques à travers le film plastique}

Il y a deux sources de déperditions thermiques vers l'atmosphère : la convection et le rayonnement dans le lointain infrarouge.

\section{Les échanges rayonnants}

Les échanges rayonnants de plus grandes longueurs d'onde, qui s'établissent entre la surface du sol et la voûte céleste, dépendent de la nature du film et de la forme de la condensation: un film de polyéthylène sec posséde une faible absorption dans les longueurs d'onde de la "fenêtre atmosphérique" (10 à $13 \mu \mathrm{m}$ ), qui permet l'essentiel du refroidissement du sol. En revanche la présence d'une faible épaisseur d'eau (quelques dizaines de $\mu \mathrm{m}$ ) suffit à bloquer ce rayonnement. Un film mouillé en gouttes réparties de façon dense ou en lame d'eau continue est donc essentiellement opaque aux infrarouge.

Dans ce cas, les pertes par rayonnement se font entre la surface du film, considéré comme un corps gris, et la voûte céleste, considérée comme un corps noir à température $T_{c}$, et s'écrivent:

$$
\varepsilon_{f} \sigma\left(T_{f}^{4}-T_{c}^{4}\right)
$$

où $T_{f}$, la température du film, et $T_{c}$, la température équivalente de la voûte céleste, sont en $\mathrm{K}$. (La constante de Stephan-Bolzman $\sigma$ vaut 5,7. 10-8).

L'émissivité $\varepsilon_{f}$ du film mouillé (supposée égale à son absorptivité dans l'infrarouge, $\alpha_{f}$ ) est proche de 0,9. La température équivalente de la voûte céleste s'exprime usuellement à l'aide d'une émissivité $\varepsilon_{c}$ de corps gris, rapportée à la température d'air au sol, sous la forme:

$$
\mathrm{T}_{c}^{4}=\varepsilon_{c} \mathrm{~T}_{a}^{4}
$$

La valeur de $\varepsilon_{c}$ dépend du contenu en humidité de l'air et de la phase (jour ou nuit) de la journée. Le modèle de Berger validé par des radiosondages (Berger, et al. 1988) permet d'obtenir une expression précise de l'émissivité du ciel, en fonction des conditions au sol (température et hygrométrie), et selon la période (jour ou nuit).

L'application des formules de Berger conduit à une émissivité équivalente de ciel comprise entre 0,80 et 0,83 dans le cas présent.

\section{Les échanges convectifs}

La convection est sensiblement indépendante de la nature du film et de la forme que prend la condensation à son contact. Elle est caractérisée par deux coefficients d'échange superficiel, qui dépendent de la nature des écoulements d'air de part et d'autre du film (convection forcée ou mixte, à l'extérieur, et convection naturelle, sous le film). II est commode d'associer à ces pertes convectives une conductance thermique $\mathrm{K}$, agissant entre l'air emprisonné sous le film et l'atmosphère.

Le calcul de cette conductance $K$ nécessite la connaissance des coefficients d'échanges convectifs de part et d'autre des films: la valeur du côté interne est assez bien définie par l'écart des températures de surface de sol et de film, puisque le moteur de la convection est l'instabilité de Rayleigh-Bénard d'une couche d'air chauffée par dessous. La corrélation de Dropkin et Sommerscales (1965) peut être utilisée pour obtenir une valeur du coefficient de Nusselt applicable à ce cas. On en déduit une valeur du coefficient d'échange convectif de la forme:

$$
h_{i} \approx 110 \mathrm{DT} / \mathrm{T}\left(\mathrm{en} \mathrm{W}^{-2} \mathrm{~m} \mathrm{~K}^{-1}\right)
$$

pour un espace entre film et sol $L=0,1 \mathrm{~m}$, une température de sol à $T(K)$ et un écart de température sol-film de DT (K); soit une valeur de $h_{i}$ qui culmine vers $10 \mathrm{~W}^{-2} \mathrm{~m} \mathrm{~K}^{-1}$ dans les conditions diurnes de l'expérience présente, et qui reste sensiblement 5 fois moins forte de nuit en raison d'un gradient très réduit.

En revanche, la convection forcée (en présence de vent), ou mixte (quand la température du film est élevée et le vent faible) met en jeu un coefficient d'échange superficiel qui dépend de la vitesse du vent. En effet, la corrélation classique de Colburn (Rubesin et Inouyer, 1973) décrivant le coefficient de Nusselt d'une plaque plane, soumise à la convection tangentielle d'un fluide en régime turbulent, donne pour une surface de film au sol sur une largeur de 2 mètres sous le vent une relation de la forme:

$$
h_{e} \approx 3 \vee 0,8 \text { en } \mathrm{W}^{-2} \mathrm{~m} \mathrm{~K}^{-1}
$$

pour une vitesse de vent $V$ donnée en $\mathrm{m} / \mathrm{s}$, donc des valeurs de $h_{e}$ comprises entre 10 et $15 \mathrm{~W}^{-2} \mathrm{~m} \mathrm{~K}^{-1}$ en conditions courantes.

II est nécessaire d'exprimer le flux $\phi$ atm de pertes thermiques du sol à travers le film polymère en fonction de la température de sol et de la température d'air extérieur. On obtient:

$$
\phi_{\text {atm }}=\left(\mathrm{K}_{e} \mathrm{~T}_{s}-\mathrm{K}_{e}^{\prime} \mathrm{T}_{a}\right) \mathrm{K}_{i} /\left(\mathrm{K}_{i}+\mathrm{K}_{e}\right)
$$

avec les notations $\mathrm{K}_{i}=\mathrm{h}_{i}+4 \varepsilon_{f} \varepsilon_{s} \sigma \mathrm{T}^{3}{ }_{s f}$

$$
\begin{aligned}
& \mathrm{K}_{e}=\mathrm{h}_{e}+4 \varepsilon_{f} \sigma \mathrm{T}^{3}{ }_{a f} \\
& \mathrm{~K}_{e}^{\prime}=\mathrm{h}_{e}+4 \varepsilon_{f} \varepsilon_{c}^{1 / 4} \sigma \mathrm{T}^{3}{ }_{a f}
\end{aligned}
$$


l'indice a désignant l'air, l'indice $f$, le film, et l'indice $s$, le sol.

Dans ces expressions, les $T_{\alpha \beta}$ représentent des valeurs moyennes entre les températures $T_{\alpha}$ et $\mathrm{T}_{\beta}$ définies comme:

$$
4 T_{\alpha \beta}^{3}=T_{\alpha}^{3}+3 T_{\alpha}^{2} T_{\beta}+3 T_{\alpha} T_{\beta}^{2}+T_{\beta}^{3}
$$

\section{La conduction de chaleur vers le sol en pro- fondeur}

Pour calculer le flux de chaleur $\phi_{s o l}$, échangé entre la surface du sol et la profondeur $z$, à la suite d'une succession de journées climatiquement analogues, il est commode de faire appel à la solution analytique correspondant à la réponse d'un milieu semi-infini et homogène, sous l'effet d'une perturbation sinusoïdale en surface. On obtient ainsi la température du sol à la profondeur $z$ (en $m$ ) comme l'image, amortie et déphasée, du signal de surface d'amplitude $T_{1}\left(e{ }^{\circ} \mathrm{C}\right.$ ) autour d'une valeur moyenne $\mathrm{T}_{0}$ (Schneider, 1973) :

$$
\mathrm{T}(z, t)=\mathrm{T}_{0}+\mathrm{T}_{1} \exp (-k z) \sin (\Omega t-k z)
$$

où $t$ est le temps (en $s$ ), $\Omega$ la pulsation du signal (en $\mathrm{s}^{-1}$ ) et où $k$, facteur d'amortissement (en $\mathrm{m}^{-1}$ ), est relié à la diffusivité thermique $\alpha\left(\mathrm{en} \mathrm{m}^{2} \mathrm{~s}^{-1}\right)$ et à la conductivité thermique $\lambda$ du sol (en $W \mathrm{~m}^{-1} \mathrm{C}^{-1}$ ) par les équations:

$$
k=\sqrt{(\Omega / 2 \alpha)}=\sqrt{(\Omega \rho C p / 2 \lambda)}
$$

$\rho$ étant la masse volumique (en $\mathrm{kg} / \mathrm{m}^{3}$ ) et $\mathrm{C} p$ la chaleur massique du sol (en J.kg-1 $\left.{ }^{\circ} \mathrm{C}^{-1}\right)$.

En présence d'un flux conductif monotone supplémentaire (et constant à l'échelle d'une journée), dû simultanément à la variation saisonnière des températures et à l'effet d'échelon créé par la pose du film sur le sol, le flux de chaleur total $\phi_{\text {sol }}$ s'écrit:

$$
\phi_{\text {sol }}=\lambda \beta-\sqrt{2} \lambda k \mathrm{~T}_{1} \cos (\Omega t-3 \pi / 4)
$$

où $\beta$ est le gradient de température moyenne journalière dans la direction $z$.

Pour connaître la réponse à un signal thermique superficiel périodique mais non sinusoïdal, il suffit de traiter chaque terme de sa décomposition en série de Fourier, et d'en déduire le flux $\phi_{\text {sol }}$ comme une série de termes harmoniques de pulsations multiples de la valeur fondamentale $\Omega$.

Deux remarques se dégagent de cette analyse:

- la composante oscillante du flux de chaleur conduit vers la profondeur est proportionnelle au produit $k \lambda$, donc à la racine carrée du terme $[\rho \mathrm{Cp} \lambda$ ) (effusivité du sol); elle est donc augmentée lors d'un accroissement de $\rho$, de $\mathrm{C} p$ ou de $\lambda$, et, en particulier, par accroissement simultané de ces trois termes lors d'un accroissement d'humidité du sol.

- la position en profondeur d'un isotherme donné est_proportionnelle au facteur $k$, c'est-à-dire à $1 / \sqrt{\alpha}$. On en déduit qu'une augmentation de la diffusivité est favorable à l'obtention de températures élevées en profondeur; mais cela ne veut pas dire qu'une augmentation d'humidité du sol aille dans ce sens, car un taux d'humidité croissant augmente à la fois la conductivité $\lambda$ et la masse volumique $\rho$.

La variation de la diffusivité du sol avec son contenu en humidité est, en effet, mise en évidence dans la Figure 9, qui représente les valeurs de $\alpha$ déduites par analyse de Fourier à diverses profondeurs et en début d'expérience: l'examen du contenu en eau d'échantillons de sol montre que les zones d'humidité maximale (en profondeur) correspondent à des valeurs de $\alpha$ minimales. Cela pouve que l'accroissement du produit $\rho \mathrm{C} \rho$ l'emporte sur celui du terme $\lambda$. En conséquence, dans le cas présent d'un sol relativement sableux, ce n'est pas en augmentant le contenu en humidité du sol que l'on va assurer une plus forte oscillation de la température de ce sol en profondeur. Seule la composante monotone du flux conductif peut contribuer, à plus long terme, à une augmentation du niveau de température moyen d'un sol humide et conducteur.

L'évolution des courbes de la diffusivité au cours du temps contient d'autres renseignements: on met en évidence un dessèchement progressif des couches superficielles au cours des 8 premiers jours, avant que soit atteint un palier stable. A ce stade, la surface du sol se trouve nettement plus sèche que les couches profondes. Les transferts d'humidité, qui ont été alternativement dirigés vers la surface interne du film (avec écoulement vers les bords) et vers la profondeur du sol, en sont responsables.

Ces transferts de masse sont, en fait, un mode de transfert thermique puissant qui vient

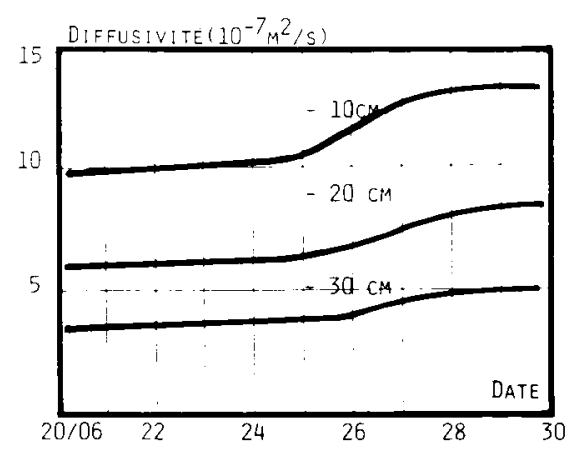

Fig. 9. Variation de la diffusivité du sol sous paillage, en fonction du temps, pour différentes profondeurs, en début d'expérience de solarisation; on constate que la perte d'humidité de la surface du sol se traduit par une augmentation progressive de la diffusivité, jusqu'à un palier stable. 
en complément du mode conductif décrit jusqu'ici. C'est donc un mécanisme qui peut contribuer utilement à la montée en température du sol en profondeur, si toutefois il reste en action sur une longue durée. Cela impose alors de mettre en place un système d'irrigation sous le paillage plastique. C'est ce qui a été fait par Naot et al. (1987), avec des résultats nuancés, qui confirment l'existence des effets antagonistes à court terme et à long terme, évoqués précédemment.

En raison de l'hétérogénéité de la teneur en humidité du sol, on peut s'interroger sur la validité du modèle analytique de Fourier adopté ici: en fait, la variation de la diffusivité $\alpha$ reste modérée, comparée à la variation de la conductivité $\lambda$ proprement dite, et la méthode de résolution reste capable d'approcher simplement les fluctuations thermiques d'un sol hétérogène. C'est en réalité l'évolution à long terme de la température en profondeur qui dépend de la conductivité $\lambda$. On a reproduit ici l'effet de la variation de cette conductivité en déduisant le coefficient $\beta$ du gradient expérimental observé pour la température moyenne journalière dans le sol à la période considérée.

\section{Le bilan énergétique global}

Les expressions des différents flux thermiques en présence permettent de modéliser la température de surface du sol, suivant la qualité optique du film polymère et suivant la période de l'année considérée. II suffit d'égaler les flux entrants et sortants en fonction du temps:

$$
G(\Theta) \tau(\Theta) A_{s o l}=\phi_{\text {atm }}+\phi_{\text {sol }}
$$

où $A_{\text {sol }}$ représente le coefficient d'absorption optique du sol et $\tau$ la transmission optique du film.

Cette équation détermine l'évolution de $T_{s}$, qui apparaît linéairement dans $\phi_{\text {sol }}$ et $\phi_{\text {atm }}$, une fois que l'on a obtenu, par procédure itérative, la convergence des paramètres $\mathrm{K} i$ et $\mathrm{Ke}$, dépendant eux-mêmes des températures.

Application: les données climatiques qui sont introduites dans le modèle, sont:

- le facteur de trouble solaire, avec éventuellement un coefficient de nébulosité de la journée considérée,

- la température de l'air sous abri et l'hygrométrie relative,

- le profil vertical de la température moyenne journalière dans le sol.

Les équations qui donnent la varation de $T_{s}$ en fonction du temps et de la profondeur, et qui résultent du bilan des flux décrits plus haut, ont fait l'objet d'un traitement sur ordinateur, à l'aide d'un programme numérique rédigé en Pascal. Seules les cinq premières harmoniques de la série de Fourier décrivant la variation périodique de $\mathrm{T}_{s}$ ont été introduites, ce qui permet néanmoins une précision suffisante. Ce programme numérique permet d'obtenir une description cohérente du profil de température de sol, au bout d'une trentaine d'itérations. L'utilisation qui en est faite ici consiste à reproduire les données expérimentales en un jour donné, pour un état donné du film, et à examiner les implications d'un tel ajustement sur la valeur des paramètres essentiels du modèle (conductivité thermique du sol, absorption optique et émissivité infrarouge des sondes de surface, coefficients d'échanges convectifs internes et externes). Puis, toutes ces valeurs étant validées et maintenues constantes, le test essentiel consiste à déduire la valeur de la température de surface sous un film dont le caractère de mouillabilité a changé.

\section{RÉSULTATS DE LA MODÉLISATION NUMÉ- RIQUE DU COMPORTEMENT DU SOL}

La simulation numérique du comportement du sol sous paillage transparent a été faite avec les paramètres climatiques et physiques détaillés dans le Tableau I.

La Figure 10a montre le résultat de la modélisation pour la période préliminaire de la solarisa-

Tableau I. Paramètres physiques introduits dans la simulation numérique de la température du sol suivant la période considérée.

Période considérée

fin juin 3 août 4 août

\begin{tabular}{|c|c|c|c|}
\hline $\begin{array}{l}\text { Coef. de trouble } \mathrm{TL} \\
\text { Coef. de nébulosité }\end{array}$ & $\begin{array}{l}5 \\
0,9\end{array}$ & $\begin{array}{l}5 \\
0,9\end{array}$ & $\begin{array}{l}5 \\
0,7\end{array}$ \\
\hline Temp. air moyenne $\mathrm{T}_{a}\left({ }^{\circ} \mathrm{C}\right)$ & 23 & 25 & 25 \\
\hline Amplitude var. $T_{a}\left({ }^{\circ} \mathrm{C}\right)$ & \pm 4 & \pm 4 & \pm 4 \\
\hline Temp. rosée moy. $T_{r}\left({ }^{\circ} \mathrm{C}\right)$ & 19 & 21 & 21 \\
\hline Amplitude var. $T_{r}\left({ }^{\circ} \mathrm{C}\right)$ & \pm 2 & \pm 2 & \pm 2 \\
\hline Hygrométrie moy. HR (\%) & 78 & 78 & 78 \\
\hline $\begin{array}{l}\text { Amplitude var. HR (\%) } \\
\text { Coef. éch. conv. } h\end{array}$ & \pm 5 & \pm 5 & \pm 5 \\
\hline $\begin{array}{l}\left(W^{-2} \mathrm{~m}^{\circ}{ }^{\circ} \mathrm{C}^{-1}\right) \\
\text { Coef. éch. conv. } h_{e}\end{array}$ & 5 & 5 & 5 \\
\hline $\begin{array}{l}\left(\mathrm{W}^{-2} \mathrm{~m}^{\circ} \mathrm{C}^{-1}\right) \\
\text { Grad. temp. Moy. sol } \beta\end{array}$ & 10 & 10 & 20 \\
\hline $\begin{array}{l}\left({ }^{\circ} \mathrm{C} / \mathrm{m}\right) \\
\text { Conduct. sol surface I }\end{array}$ & 50 & 0 & 0 \\
\hline $\begin{array}{c}\left(W^{-2} \mathrm{~m}^{\circ} \mathrm{C}^{-1}\right) \\
\text { Coef amortiss } k\left(\mathrm{~m}^{-1}\right)\end{array}$ & ${ }_{12}^{0,15}$ & 0,15 & 0,15 \\
\hline Coef. amortiss. $k\left(m^{-1}\right)$ & 12 & 12 & \\
\hline
\end{tabular}



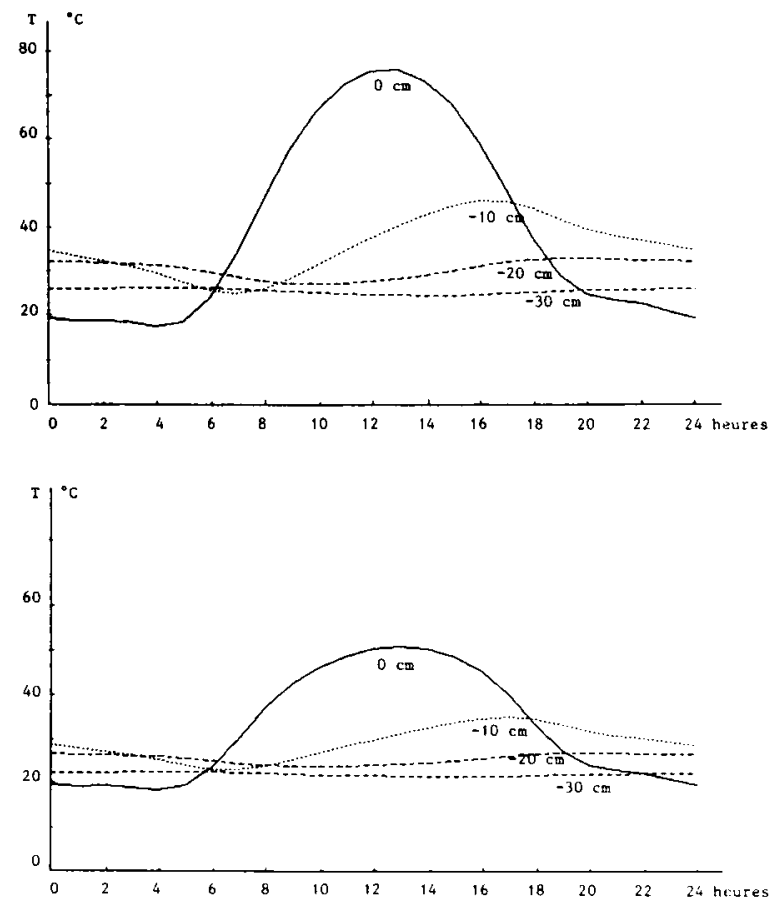

Fig. 10. Résultat de la modélisation de la température du sol sous paillage avec les conditions climatiques de la journée du 20 juin 1985. En 10a, les températures calculées en surface et en profondeur sous un film intégralement mouillé sont très élevées, conformément à l'expérience; en 10b, la simulation des températures correspondantes sous un film supposé non mouillable donne des valeurs fortement réduites.

tion (conditions climatiques de fin juin 1985), pour un film mouillable: des maximums de $75^{\circ} \mathrm{C}$ en surface de sol impliquent l'existence d'une couche superficielle de terre peu conductrice $\left(\lambda \approx 0,15 \mathrm{~W} \mathrm{~m}^{-1} \mathrm{~K}^{-1}\right)$ et de faible densité $(\rho \approx$ $500 \mathrm{~kg} / \mathrm{m}^{3}$ ), dont la diffusivité $\alpha$ reste néanmoins peu différente de celle rencontrée en profondeur $\left(\alpha \approx 3.010^{7} \mathrm{~m} / \mathrm{s}\right)$. Une telle couche poreuse et isolante existe effectivement et résulte d'une dessication importante de la couche superficielle du sol sous l'effet du rayonnement intense. Les paramètres physiques étant fixés, la perte éventuelle de mouillabilité du film, simulée en Figure $10 \mathrm{~b}$, se traduirait par une réduction de la température de plus de $20^{\circ} \mathrm{C}$.

La Figure 11a montre le résultat de la simulation obtenu pour les conditions climatiques du 3 août 1985, journée pendant laquelle les températures ambiantes sont plus élevées qu'en juin et le flux conductif vers la profondeur du sol insignifiant. Le film, qui est encore mouillé d'une couche d'eau continue, permet d'obtenir des températures aussi élevées qu'en début d'expérience, malgré une trajectoire du soleil moins favorable. Le 4 août est caractérisé par une légère baisse du rayonnement et des échanges convectifs plus intenses, tandis que le film perd sa mouillabilité. Le modèle confirme (Fig. 11b) la chute spectaculaire des températures de surface
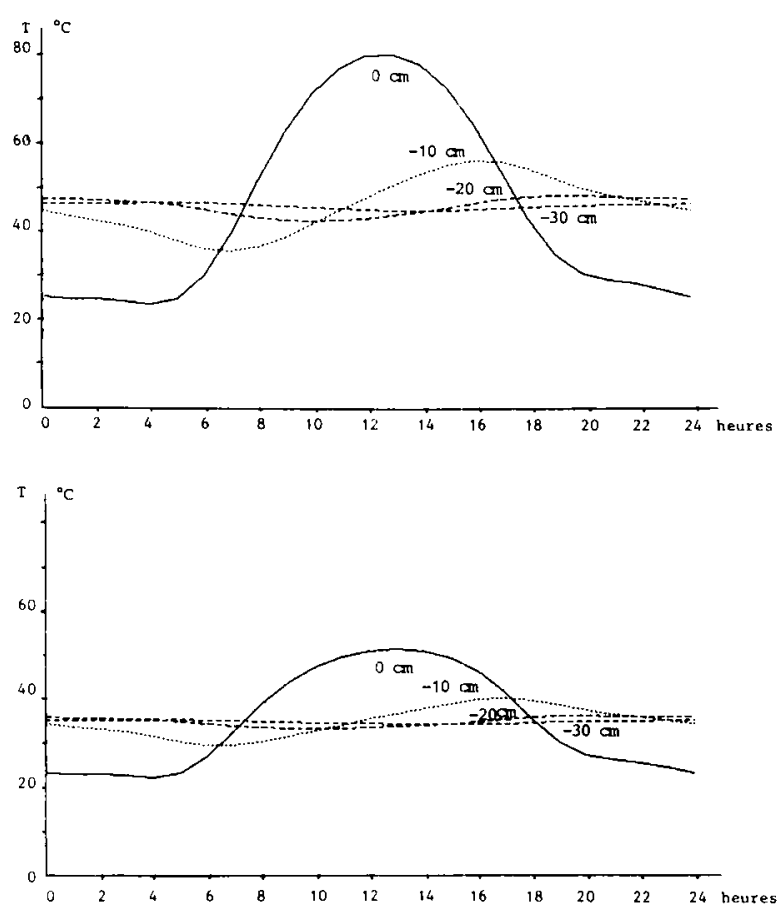

Fig. 11. Résultats de la modélisation pour des journées d'août 1985. En 11a, le film est supposé mouillable, et avec les conditions climatiques du 3 août, les températures calculées sont très élevées; en $11 \mathrm{~b}$, le film est non mouillable, les conditions sont celles du 4 août et les températures sont fortement réduites.

de sol (une baisse de $28^{\circ} \mathrm{C}$ du maximum journalier).

Enfin, la modélisation permet de proposer une interprétation du décalage observé entre les sondes de température de surface des deux parcelles bâchées: il suffit qu'une sonde se trouve placée au dessus du sol, et non plus à son contact, pour qu'elle donne une réponse surestimée de $5^{\circ} \mathrm{C}$; mais, mieux encore, si l'émissivité dans l'infrarouge d'une des sondes se trouve réduite d'une valeur de 0,9 à une valeur de 0,7 , la surestimation peut atteindre $10^{\circ} \mathrm{C}$; or, un effet de sélectivité inhabituel, consécutif à l'existence d'une couche d'oxyde particulièrement fine sur la surface du cuivre de la sonde, n'est pas à exclure.

L'influence prépondérante de la transmission optique d'un film affecté de condensation sur les températures d'un sol sous bâche est donc confirmée par le calcul. Les températures très élevées, relevées en début de période, sont également dues au fait que la couche superficielle du sol a joué un rôle d'isolant et a limité la transmission de la chaleur vers la profondeur. La perte brutale de mouillabilité du film souligne de façon spectaculaire le rôle de sa transmission optique sur le comportement thermique du sol. Mais la chute de température est suffisamment tardive pour ne pas compromettre le résultat de la désin- 
fection finale, comme cela est indiqué ci-dessous.

\section{RÉSULTATS AGRONOMIQUES ET BIOLO- GIQUES SUR CULTURE D'ANÉMONES}

\section{Contamination par l'inoculum tellurique de la mycose}

Dans toutes les parcelles étudiées, la culture d'anémones mise en place s'est révélée pratiquement indemne de contamination

\section{Développement de la végétation adventice}

Le dénombrement approximatif des mauvaises herbes est résumé dans le diagramme de la Figure 12, qui visualise les moyennes sur 4 répétitions. On observe que la parcelle témoin est la plus enherbée, suivie par la parcelle traitée (80 g/m² de $\mathrm{CH}_{3} \mathrm{Br}$ ). Les deux parcelles recouvertes de paillis plastique transparent procurent la meilleure éradication (sans effet évident du traitement au $\mathrm{CH}_{3} \mathrm{Br}$ à demi-dose).

\section{Analyse nématologique du sol}

Deux séries d'analyses nématologiques successives ont été pratiquées dans l'ensemble des

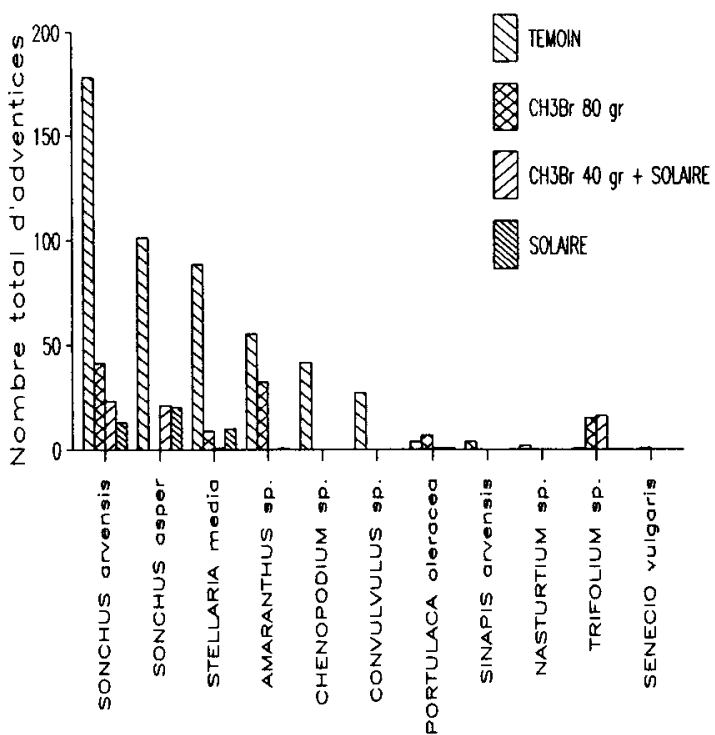

Fig. 12. Dénombrement approximatif des différentes sortes d'adventices qui se sont développées lors de la période de solarisation sur les différentes parcelles.

parcelles étudiées, et ce à différentes profondeurs: l'une au moment de l'enlèvement des paillis plastiques (au 30 août), et l'autre en fin de culture d'anémones (le 7 novembre). Les Tableaux 2 et 3 , qui regroupent les moyennes effectuées sur 4 répétitions, comptabilisent les résultats observés en nombre d'individus.

Tableau II. Répartition des nématodes par groupes trophiques dans l'analyse effectuée à l'issue de la période de solarisation (moyenne de 4 répétitions).

\begin{tabular}{lrrrrrrrr}
\hline \multirow{2}{*}{$\begin{array}{l}\text { PARCELLE } \\
\text { Profondeur }(\mathrm{cm})\end{array}$} & \multicolumn{2}{c}{ TÉMOIN } & \multicolumn{2}{c}{$\mathrm{CH}_{3} \mathrm{Br} 80 \mathrm{~g} / \mathrm{m}^{2}$} & \multicolumn{2}{c}{ FILM EP } & \multicolumn{2}{c}{$E P+\mathrm{CH}_{3} \mathrm{Br} 4 \mathrm{Og} / \mathrm{m}^{2}$} \\
& $0-5$ & $20-25$ & $0-5$ & $20-25$ & $0-5$ & $20-25$ & $0-5$ & $20-25$ \\
\hline Bactériophages & 982 & 378 & 86 & 31 & 9 & 187 & 2 & 33 \\
Créophages & $<1$ & 4 & 0 & 0 & 0 & 0 & 0 & 0 \\
Mycophages & 37 & 3 & 2 & 2 & 2 & $<1$ & $<1$ & $<1$ \\
Omnivores & 0 & 0 & 0 & 1 & 0 & $<1$ & 0 & 0 \\
Phytophages & 166 & 14 & 13 & 4 & 2 & 5 & $<1$ & $<1$ \\
Pop. totale & 1185 & 399 & 101 & 38 & 13 & 192 & 2 & 33 \\
\hline
\end{tabular}

Tableau III. Répartition des nématodes par groupes trophiques dans l'analyse effectuée à l'issue de la culture d'anémones (moyenne de 4 répétitions).

\begin{tabular}{|c|c|c|c|c|c|c|c|c|c|c|c|c|c|c|c|c|}
\hline \multicolumn{2}{|c|}{ PARCELLE } & \multicolumn{3}{|c|}{ TÉMOIN } & \multirow[b]{2}{*}{5} & \multicolumn{3}{|c|}{$\mathrm{CH}_{3} \mathrm{Br} 80 \mathrm{~g} / \mathrm{m}^{2}$} & \multirow[b]{2}{*}{5} & \multicolumn{2}{|c|}{ FILM EP } & \multirow[b]{2}{*}{35} & \multirow[b]{2}{*}{5} & \multicolumn{3}{|c|}{$\mathrm{EP}+\mathrm{CH}_{3} \mathrm{Br} 4 \mathrm{Og} / \mathrm{m}^{2}$} \\
\hline $\mathrm{cm}$ & 5 & 15 & 25 & 35 & & 15 & 25 & 35 & & 15 & 25 & & & 15 & 25 & 35 \\
\hline B. & 362 & 244 & 110 & 83 & 587 & 164 & 82 & 43 & 433 & 206 & 84 & 56 & 164 & 113 & 41 & 23 \\
\hline c. & $<1$ & 1 & 0 & 1 & 0 & 0 & 0 & 0 & 1 & 1 & $<1$ & $<1$ & 0 & 0 & 0 & $<1$ \\
\hline M. & 57 & 12 & 4 & 4 & $<1$ & 1 & 0 & 0 & 5 & 2 & $<1$ & 0 & 5 & 0 & 0 & 0 \\
\hline O. & 11 & 3 & 3 & 3 & 0 & 0 & 0 & 0 & 3 & 2 & 2 & $<1$ & $<1$ & 0 & 0 & 0 \\
\hline P. & 47 & 7 & 1 & 1 & 0 & 2 & 0 & 0 & 2 & 0 & 1 & 0 & 0 & $<1$ & $<1$ & 0 \\
\hline$\Sigma$ & 477 & 267 & 118 & 92 & 587 & 167 & 82 & 43 & 444 & 211 & 87 & 56 & 169 & 113 & 41 & 23 \\
\hline
\end{tabular}


On constate une hiérarchie bien établie des effectifs de populations de nématodes pour les différentes parcelles à l'issue de la phase de solarisation: la plus pauvre en nématodes résiduels étant, naturellement, la parcelle paillée et traitée à demi-dose de $\mathrm{CH}_{3} \mathrm{Br}$, la parcelle paillée et non traitée prenant l'avantage sur les parcelles nues, à faible profondeur. En revanche, on voit que le biocide gazeux est capable de toucher des profondeurs que n'atteint pas le traitement thermique solaire, en raison notamment d'une trop faible diffusivité thermique du sol. Enfin, la solarisation s'avère plus active contre les nématodes localisés dans les couches superficielles que la désinfection au bromure de méthyl.

A l'issue de la période de culture, les effets sont moins contrastés en terme de population globale, l'avantage restant au couplage du paillage et du traitement chimique. L'effet principal est la réduction des phytophages à faible profondeur par effet thermique, aussi bien que par effet chimique, ainsi que la remontée de la population de bactériophages à toute profondeur et dans tous les cas de figure.

\section{CONCLUSION}

Des températures remarquablement élevées, obtenues en surface de sol, sous un film mouillable, ont contribué à la qualité de la désinfection du sol par voie solaire. Même aux profondeurs où la solarisation ne permet pas d'atteindre les températures léthales nécessaires à l'élimination complète des organismes indésirables, les modifications qu'elle engendre dans les biocénoses telluriques aboutissent à un bilan favorable pour la conduite de la culture. L'usage de films doués d'une bonne transmission optique dans les longueurs d'ondes du visible et du proche infrarouge, et d'une bonne opacité dans le domaine des grandes longueur d'onde, est un élément décisif dans l'optimisation du processus de désinfection par voie solaire, notamment sous les latitudes à climat tempéré. L'effet favorable d'une forte mouillabilité des films est clairement démontrée. II convient de noter que les films anti-buée actuels ont une propriété de mouillabilité très limitée dans le temps: la durée de fonctionnement de l'agent mouillant peut aller d'une semaine à quelques semaines, suivant la rapidité de lavage de l'agent tensio-actif par les condensats. Cette rapidité dépend de l'humidité disponible dans la couche superficielle du sol et de facteurs climatiques, comme le vent.

Le manque de conductivité des couches superficielles du sol reste néanmoins une limitation à l'élévation de température du sol en pro- fondeur. Ce défaut pourrait être corrigé par une opération préliminaire de tassement du sol et par une meilleure exploitation des transferts de masse au sein du sol: une quantité de vapeur d'eau supplémentaire, créée périodiquement par des arrosages du sol sous le paillage à des moments soigneusement choisis, pourrait ainsi contribuer à réchauffer le sol en profondeur pendant une durée prolongée à volonté.

Dans le cas présent, la solarisation, efficace pendant les 45 premiers jours, grâce à la mouillabilité prolongée du film, et associée à un traitement biocide réduit, apparaît comme un compromis permettant d'assurer un état sanitaire du sol acceptable, sans dépeuplement bactérien excessif et, avec un risque de résidus chimiques atténué.

\section{ANNEXE}

\section{Trajet des rayons d'incidence oblique sur un dioptre hémisphérique.}

\section{Le schéma est celui de la Figure 13.}

On peut voir qu'une fois le dioptre plan $(\mathrm{P})$ franchi sous incidence oblique $\Theta_{i}$, les rayons, alors inclinés d'un angle $\Theta_{r}$, rencontrent un dioptre hémisphérique non distinguable de celui tracé en pointillé, et ayant pour base le plan $\left(P^{\prime}\right)$ incliné de $\Theta_{r}$ par rapport à $(P)$, ce qui ramène l'étude au cas de la figure 6 . En conséquence, le lieu des points d'incidence se traduisant par une transmission du rayonnement à travers le dioptre hémisphérique est la surface de l'ellipse du plan (P) qui se projette orthogonalement sur le plan $\left(P^{\prime}\right)$ sous forme du cercle de rayon $R / n$.

Cette ellipse a donc pour petit axe $R / n$ et pour grand axe $\mathrm{R} /\left(n \cos \Theta_{r}\right)$ et son aire est donc

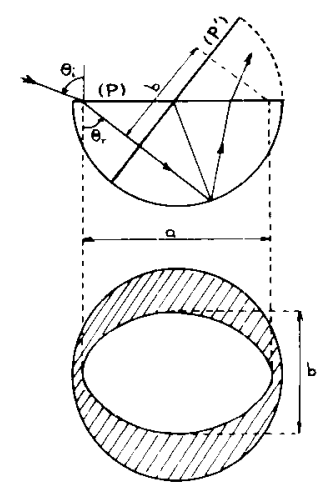

Fig. 13. Trajet optique d'un rayon d'incidence oblique $\Theta_{i}$ sur le plan diamètral d'un milieu optique hémisphérique. Le trajet se ramène à celui d'un rayon en incidence normale sur un milieu optique hémisphérique basculé d'un angle $\Theta_{r}$ par rapport au précédent. La couronne circulaire (de rayon $b$ ) qui définit la zone de réflexion totale en incidence normale se projette en une ellipse d'axes $a$ et $b$ (avec $a>b$ ). 
$\pi \mathrm{R}^{2} /\left(n^{2} \cos \Theta_{r}\right)$

qui peut enfin s'exprimer en fonction de $\Theta_{i}$ grâce à la relation de Descartes

$$
\sin \Theta_{i}=n \sin \Theta_{r}
$$

sous la forme

$$
\pi \mathrm{R}^{2} /\left[n \sqrt{\left(n^{2}-\sin ^{2} \Theta_{i}\right)}\right]
$$

ce qui se traduit par une augmentation de la surface, donnant lieu à transmission du rayonnement, par rapport au cas de l'incidence normale, d'un facteur $n \sqrt{\left(n^{2}-\sin ^{2} \Theta_{i}\right)}$ pouvant atteindre $152 \%$.

\section{Calcul de la transmission globale d'un film affecté d'une condensation en gouttes}

On considère d'abord le passage à travers les deux dioptres plans (air-film) puis (film-eau), ce qui permet d'avoir un premier effet de l'angle d'incidence sur la transmission optique. Puis on analyse le cheminement des rayons à travers le milieu aqueux, en simplifiant l'effet des réflexions partielles au sein de ce milieu plan-convexe: on considère que le coefficient de réflexion partielle affectant les rayons qui frappent la sphère du côté interne est constant et égal à celui obtenu pour un angle $\Theta_{0}=30^{\circ}\left(\Theta_{0}\right.$ est sensiblement la moyenne entre les angles d'incidence limites $0^{\circ}$ et $\left.\Theta_{\max }=\arcsin (1 / n)\right)$, que ces rayons peuvent prendre.

D'autre part, les rayons qui subissent une réflexion totale sur la sphère interne conservent, après réflexion partielle sur le dioptre plan supérieur, une incidence inchangée sur le dioptre sphérique et ne seront donc jamais transmis vers le bas.

En conséquence, l'expression simplifiée obtenue pour le coefficient de transmission d'un film supportant un réseau dense de gouttes de condensation d'écrit:

$$
T(\Theta)=\frac{\left[1-\rho_{12}(\Theta)\right]\left[1-\rho_{23}(\Theta)\right]\left[1-\rho_{13}\left(\Theta_{0}\right)\right]}{\left[1-\rho_{12}(\Theta) \rho_{23}(\Theta)\right] n_{3}\left[n_{3}^{2}-\sin ^{2}(\Theta)\right]^{1 / 2}}
$$

où les indices 1,2 et 3 désignent les milieux respectifs de l'air, du polymère et de l'eau.

\section{RÉFÉRENCES}

Avissar R., Mahrer Y., Margulies L., Katan J. (1986) Field aging of transparent polyethylene mulches; I \& II; Soil Sc. Soc. Am. J. 50, (1), 205-209

Berger X., Cubizolles B. \& Donet I. (1988) Radio-sounding data for the determination of the infra-red sky radiation; Solar \& Wind Technology, 5, (4), 353-363
Berninger E., Pionnat J.C., \& Scotto La Massese C. (1985) Essai de désinfection solaire des sols dans le sud-est de la France. Agronomie 5, 505-513

Braun M., Koch W., Stiefvater M. (1987) Solarisation for soil sanitation - possibilities and limitations. Demonstrated in trials in southern Germany and the Sudan. Gesunde Pflanzen 39, (7), 301-309

Dropkin \& Sommerscales (1965) Heat transfer by natural convection in a liquid confined by 2 parallel plates which are inclined at various angles with respect to horizontal. Heat Transfers $87,77-84$

Duffie J. A. \& Beckman W.A. (1974) Solar Energy Thermal Processes; John Wiley \& Sons Inc.; Wiley Interscience, pp. 15 \& 108

Garibaldi A. (1983) Attempts to use soil solarisation closed greenhouses in northern Italy for the control of soil-born pathogens of tomato. 2nd Int. Symp. Soil Disinfect. Leuven (Belgium), 46

Goisque M.J., Louvet H., Martin C., Lagier J., Davet P., Couteaudier Y., Louvet J. (1984) La désinfection solaire du sol. Un moyen de lutte contre la maladie des racines liègeuses de la tomate. Rev. Hortic., P.H.M., 247, 49

Jaffrin A. \& Venard M. (1983) Stockage thermique et doublage de parois dans les serres solaires. Plasticulture, 52, 7

Johnson A.W., Jaworski C.A., Glaze N.C., Summer D.R., Chalfant R.B. (1981) Effect of film mulch and soil pesticides on nematodes, weeds and yield vegetable crops. J. Nematol. 13, 141

Kassaby F.Y. (1985) Solar-heating soil for control of damping-off diseases. Soil Biol. Biochem. 17, (4), 429434

Katan J., Greenberger A., Alan H. \& Grinstein H. (1976) Solar heating by polyethylene mulching for the control of diseases caused by soil-borne pathogens. Phytopathology 66, 683

Katan J., Grinstein A., Greenberger A., Yarden O. \& Devay J.E. (1987) The first decade (1976-1986) of soil solarization (solar heating): a chronological bibliography. Phytoparasitica 15, (3), 229-255

Kye U.K., Kim K.C (1985) Possibility of soil solarization in Korea. Kor. J. of Plant Protec.; 24, (2), 107-114

Malathrakis N.E., Kapetanis G.E. \& Linardakis (1983) Brown root rot of tomato and its control in Crete. Ann. Appl. Biol. 102, 251

McSorley R. \& Parrado J.L. (1986) Application of soil solarisation to rockdale soils in a subtropical environment. Nematropica 16, (2), 125-140

Naot O., Mahrer Y., Rawitz E., Avissar R. \& Katan J. (1987) The effect of reirrigation on the thermal regime of polyethylene-mulched soils: experimental and numerical studies. Soil Sci. 144, (2), 101-106

Perrin de Brichambaut C. \& Vauge C. (1982) Le Gisement Solaire. Lavoisier. Tech. \& Doc.

Rubesin M.W. \& Inouyer M. (1973) Forced convection, external flow. In'. Handbook of Heat Transfer; Chap. 8, p. 11; (W.M. Rohsenow \& J.P. Hartnett eds.) McGraw Hill Book

Schneider P.J. (1973) Conduction. In : Handbook of Heat Transfer, Chap 3, pp. 37-70. (W.M. Rohsenow \& J.P. Hartnett,eds.); McGraw Hill Book Co

Scotto La Massese C. et al. (1985) Expérimentation de divers films plastiques pour une solarisation de sol de culture. Rapport interne; Laboratoire de recherche de nématologie, INRA, 123 Bld F. Meilland BP 2079 06606 Antibes Cedex 